\title{
Ohmic contact formation on n-type Ge
}

\author{
R.R. Lieten \\ III-V Systems, IMEC, Kapeldreef 75, 3001 Leuven, Belgium \\ ETRO, Vrije Universiteit Brussel, Pleinlaan 2, 1050 Brussels, Belgium \\ S. Degroote \\ III-V Systems, IMEC, Kapeldreef 75, 3001 Leuven, Belgium \\ M. Kuijk \\ ETRO, Vrije Universiteit Brussel, Pleinlaan 2, 1050 Brussels, Belgium \\ G. Borghs \\ NEXT, IMEC, Kapeldreef 75, 3001 Leuven, Belgium
}

\section{Abstract}

Severe Fermi level pinning at the interface between n-Ge and a metal, leads to the formation of a Schottky barrier, almost independent on the metal work function. Therefore it seems impossible to form metal Ohmic contacts on moderately, n-type doped Ge layers. For p-type Ge the Fermi level pinning works opposite: all metal contacts show Ohmic behavior. This fixed behavior can be altered by the introduction of a thin $\mathrm{Ge}_{3} \mathrm{~N}_{4}$ layer. $\mathrm{Ge}_{3} \mathrm{~N}_{4}$ seems effective in reducing Fermi level pinning and therefore allows the formation of Ohmic contacts on n-type $\mathrm{Ge}$ and a rectifying contact on p-type Ge. 
Germanium is an important semiconductor material, mainly used in transistors and integrated circuits. A metal-oxide-semiconductor field effect transistor (MOSFET) with Ge channel currently receives much attention as a possible candidate for next-generation high mobility devices, because of the high electron and hole mobility of Ge as compared to Si. However many problems concerning Ge still need to be solved. One problem is the formation of low resistance contacts. Severe Fermi level pinning at the interface between n-Ge and a metal, leads to the formation of a Schottky barrier, almost independent on the metal work function [1]. For Ge n-channel MOSFET devices the lack of low resistance Ohmic contacts leads to high resistance source and drain contacts, limiting the obtainable channel current. The formation of Ohmic contacts on n-type Ge is therefore an important step towards realizing n-MOSFETs in Ge. For p-type Ge the Fermi level pinning works the opposite: all metal contacts show Ohmic behavior. In theory, the fabrication of low resistance contacts on semiconductors is based on selecting a metal with a low work function for n-type semiconductors and a high work function for p-type semiconductors to obtain a low barrier height. In most cases however this method does not work well: interface states pin the Fermi-level and make the barrier height independent of the metal work function. To obtain low resistivity contacts a thin layer of very heavily doped semiconductor is most often used. This leads to a very thin depletion region, known as a tunnel junction, through which field emission can take place. This kind of contacts still requires low barrier heights for high current conduction. For sufficient low barrier heights the formation of a tunnel junction is not needed. Beside good conductivity, Ohmic contacts should also exhibit metallurgical stability. Silicides, stoichiometric compounds of metals and $\mathrm{Si}$, often show these properties and are consequently often used. Germanides, stoichiometric compounds of metals and Ge, are also used on Ge layers. Ti germanide contacts and sulfur-passivated Ni germanide 
contacts on $\mathrm{n}$-Ge show barrier height values as low as 0.34 [2] and $0.15 \mathrm{eV}$ [3] respectively, but current-voltage (IV) of these contacts are still clearly rectifying [3].

We investigated the influence of a thin $\mathrm{Ge}_{3} \mathrm{~N}_{4}$ layer, introduced between a metal contact and $\mathrm{Ge}$, on the current conduction. Nitridation of the Ge(001) surface in ultra high vacuum (UHV) by a nitrogen plasma cell is known to form amorphous $\mathrm{Ge}_{3} \mathrm{~N}_{4}$ at temperatures of 100$550^{\circ} \mathrm{C}[4,5]$. It was reported that after annealing at $600^{\circ} \mathrm{C}$ in vacuum this layer completely evaporated, implying that the $\mathrm{Ge}_{3} \mathrm{~N}_{4}$ film is not stable at temperatures above $600^{\circ} \mathrm{C}$. Recently we have experimentally shown that exposure of $\mathrm{Ge}(111)$ to a nitrogen plasma below $550{ }^{\circ} \mathrm{C}$ leads to the formation of an amorphous $\mathrm{Ge}_{3} \mathrm{~N}_{4}$ layer while at a temperature above $600{ }^{\circ} \mathrm{C}$ a thin, mono crystalline $\mathrm{Ge}_{3} \mathrm{~N}_{4}$ layer is formed [6]. The $\mathrm{Ge}_{3} \mathrm{~N}_{4}$ formed at high temperature is most likely $\beta$ $\mathrm{Ge}_{3} \mathrm{~N}_{4}$ which is the most stable crystalline phase of $\mathrm{Ge}_{3} \mathrm{~N}_{4}[7]$ and has a hexagonal structure. $\beta$ $\mathrm{Ge}_{3} \mathrm{~N}_{4}$ can only form an epitaxial layer on three fold symmetric $\mathrm{Ge}(111)$. On the $\mathrm{Ge}(001)$ plane, the $\mathrm{Ge}_{3} \mathrm{~N}_{4}$ that is formed with high temperature nitridation is in a polycrystalline phase, as we observed by reflection high energy electron diffraction (RHEED). The bandgap of $\beta-\mathrm{Ge}_{3} \mathrm{~N}_{4}$ has been calculated to be $3.1 \mathrm{eV}[7]$.

The experimental procedure for contact formation is described below. Ge substrates were chemically cleaned to remove metallic contamination, particles and native oxide from the surface, just before loading into an UHV system. Subsequently, annealing in vacuum ( $\sim 1$ e-9 Torr) was done to degas the samples. The cleanliness of the surface was confirmed by RHEED, which showed a reconstructed surface. On this clean Ge surface either (poly) crystalline or amorphous $\mathrm{Ge}_{3} \mathrm{~N}_{4}$ was formed by exposure to nitrogen plasma. As reference Ge substrates were prepared in the same way, but without $\mathrm{Ge}_{3} \mathrm{~N}_{4}$ formation on top. Finally, the samples were taken 
out of the UHV system and immediately loaded into a metal deposition system to suppress oxide formation. Metal contacts with a diameter of $500 \mu \mathrm{m}$ were deposited by means of a shadow mask. In order to prevent the metal from reacting with ambient air, an Au capping layer was deposited on top of the metal. As backside contact complete coverage by metal was used. Because of the large surface area, Ohmic behavior could be observed for this contact. We have repeated this experiment for different metals, substrate orientations and doping types. N-type $\mathrm{Ge}(111)$ and $\mathrm{Ge}(001)$ substrates with a carrier concentration of around $1 \times 10^{16} \mathrm{~cm}^{-3}(\sim 0.15$ Ohmcm) were used and also p-type Ge(111) substrates with a carrier concentration of around $2 \mathrm{x}$ $10^{14} \mathrm{~cm}^{-3}(\sim 15 \mathrm{Ohmcm})$. X-ray photoelectron spectroscopy measurements revealed the formation of a $\sim 0.7 \mathrm{~nm} \mathrm{Ge}_{3} \mathrm{~N}_{4}$ layer in the case of crystalline $\mathrm{Ge}_{3} \mathrm{~N}_{4}$ on $\mathrm{Ge}(111)$. No post deposition anneal was performed. IV measurements were performed between the front and backside contacts.

For the $\mathrm{Al}$ contact on $\mathrm{n}-\mathrm{Ge}(111)$ without intermediate $\mathrm{Ge}_{3} \mathrm{~N}_{4}$ a rectifying behavior was observed, as reported by others [1]. The sample with $\mathrm{Ge}_{3} \mathrm{~N}_{4}$ intermediate layer however showed Ohmic behavior with a current density of $14 \mathrm{~A} / \mathrm{cm}^{2}$ at $-1 \mathrm{~V}$, as shown in Figure 1. The lack of current saturation in reverse and the obtained current densities indicate the barrier height of the contact is smaller than $0.3 \mathrm{~V}$.

Ni germanide (NiGe) is often used to form low resistance contacts on n-Ge, with reported barrier heights ranging between 0.39 [2] and $0.61[3]$. Therefore NiGe/n-Ge(111) contacts were made as a comparison to $\mathrm{Al} / \mathrm{Ge}_{3} \mathrm{~N}_{4} / \mathrm{n}-\mathrm{Ge}(111)$ contacts. From Figure 1 and Table I it is clear that the $\mathrm{Al} / \mathrm{Ge}_{3} \mathrm{~N}_{4} / \mathrm{n}-\mathrm{Ge}(111)$ contact shows much better current conduction than $\mathrm{NiGe} / \mathrm{n}-\mathrm{Ge}(111)$. The barrier height of the NiGe/n-Ge(111) contact was calculated from the reverse current to be $0.60 \mathrm{eV}$, close to the value reported by reference 3 . 
$\mathrm{Al}$ contacts with an amorphous intermediate $\mathrm{Ge}_{3} \mathrm{~N}_{4}$ layer showed a comparable current conduction as for $\mathrm{Al}$ contacts with a crystalline $\mathrm{Ge}_{3} \mathrm{~N}_{4}$ layer. Therefore it seems that both amorphous as crystalline $\mathrm{Ge}_{3} \mathrm{~N}_{4}$ layers lead to improved current conduction for $\mathrm{Al}$ contacts on $\mathrm{n}$ $\mathrm{Ge}(111)$.

The influence of an amorphous $\mathrm{Ge}_{3} \mathrm{~N}_{4}$ and a polycrystalline $\mathrm{Ge}_{3} \mathrm{~N}_{4}$ intermediate layer for Al contacts on $\mathrm{n}-\mathrm{Ge}(001)$ was investigated. For both contacts the IV measurements showed Ohmic behavior. It was necessary to reduce the nitrogen plasma exposure time for the formation of amorphous $\mathrm{Ge}_{3} \mathrm{~N}_{4}$ on $\mathrm{Ge}(001)$ in comparison with $\mathrm{Ge}(111)$ to obtain similar behavior. This suspects that the $\mathrm{Ge}_{3} \mathrm{~N}_{4}$ thickness is larger in the case of $\mathrm{Ge}(001)$ for the same nitrogen plasma settings and exposure time. This can be explained by the denser atomic surface packing of $\mathrm{Ge}(111)$ which can limit the rate of $\mathrm{Ge}_{3} \mathrm{~N}_{4}$ formation in respect with $\mathrm{Ge}(001)$. This points to the advantage of using high substrate temperatures during plasma nitridation, because this leads to an equilibrium between formation and dissociation of $\mathrm{Ge}_{3} \mathrm{~N}_{4}$. This equilibrium limits the obtainable thickness and therefore allows accurate thickness control [6].

We have also investigated other methods than nitrogen plasma exposure in vacuum to obtain a $\mathrm{Ge}_{3} \mathrm{~N}_{4}$ layer on top of Ge. Annealing in nitrogen and ammonia atmosphere also showed improved contacts for Al.

The current conduction of different metals on $\mathrm{n}-\mathrm{Ge}(111)$ has been investigated. $\mathrm{Al}, \mathrm{Cr}, \mathrm{Co}$, $\mathrm{Au}$ and Pt contacts were used. All contacts without $\mathrm{Ge}_{3} \mathrm{~N}_{4}$ showed rectifying behavior. The insertion of a thin $\mathrm{Ge}_{3} \mathrm{~N}_{4}$ layer did not change the electrical behavior of the junction for $\mathrm{Au}$ and Pt: the IV characteristic remained clearly rectifying. For $\mathrm{Al}, \mathrm{Cr}$ and $\mathrm{Co}$ on the other hand the addition of a thin $\mathrm{Ge}_{3} \mathrm{~N}_{4}$ layer has a large influence on the IV characteristics. For these metals the 
junction has become Ohmic: high current density both in forward and reverse bias, see Figure 2. From the reverse currents the barrier height was calculated and summarized in Table II. From the IV behavior of different metal contacts it can be concluded that the introduction of a thin $\mathrm{Ge}_{3} \mathrm{~N}_{4}$ layer clearly reduces the Fermi level pinning, and therefore allows changing the barrier by choosing a metal with different work function.

At the surface of a semiconductor with covalent bonds atoms have unpaired electrons, often referred to as dangling bonds. These dangling bonds can either give up or accept an electron. The corresponding surface states may be situated in the forbidden band gap as is the case for Ge [1]. Depending on the surface states distribution and the Fermi level of the bulk, these states will be partially filled and therefore can lead to a positive or negative net surface charge. The position of the Fermi level at which the surface is electrically neutral is called the charge neutrality level. The large density of surface states of Ge will force the Fermi level to the position of the charge neutrality level, which is situated slightly above the valence band in the case of Ge [1]. An electron depletion region and consequent electron barrier are formed at the n-Ge surface. This barrier still exists when a metal is deposited and is almost independent of the metal work function. Therefore all metal contacts on $n-G e$ lead to rectifying behaviour. Amorphous $\mathrm{Ge}_{3} \mathrm{~N}_{4}$ has been shown to be a good passivation of $\mathrm{Ge}(001)$ [4]. It has also been shown by theoretical calculations that crystalline $\mathrm{Ge}_{3} \mathrm{~N}_{4}$ is a passivation layer for $\mathrm{Ge}(111)$ surfaces [7]. A thin $\mathrm{Ge}_{3} \mathrm{~N}_{4}$ layer, regardless whether it is amorphous or crystalline, will reduce the number of dangling bonds at the Ge surface and therefore the surface state density. The difference in Pauling electronegativity of nitrogen and germanium is 1.0. Therefore the Ge-N binding is not pure covalent, but partially ionic. From this polarized covalent binding, the Fermi level pinning is expected to be low [8]. Therefore the $\mathrm{Ge}_{3} \mathrm{~N}_{4}$ has a double effect: (1) it terminates dangling bonds 
at the Ge surface states and consequently reduces the trap density at the $\mathrm{Ge}_{3} \mathrm{~N}_{4} / \mathrm{Ge}$ interface; (2) it contains less surface states, and therefore the metal/ $\mathrm{Ge}_{3} \mathrm{~N}_{4}$ interface shows much less Fermi level pinning. Because $\mathrm{Ge}_{3} \mathrm{~N}_{4}$ is a wide band gap semiconductor and therefore acts as a barrier layer for electron injection, this layer should be kept thin enough so that carriers can tunnel easily through it. For p-Ge the Fermi level pinning close to the valence band is beneficial for the formation of a p-type Ohmic contact: even when a metal with work function smaller than the sum of the Ge electron affinity and band gap is deposited, an Ohmic contact is formed. If the model presented above is correct, $\mathrm{Ge}_{3} \mathrm{~N}_{4}$ passivation of $\mathrm{p}-\mathrm{Ge}$ is expected to allow the formation of Schottky barriers. Indeed we clearly observe rectifying behaviour of Al contacts on p-Ge(111) with a crystalline $\mathrm{Ge}_{3} \mathrm{~N}_{4}$ intermediate layer, see Figure 3. For $\mathrm{Cr}$ the difference between the metal work function of $\mathrm{Cr}(4.5 \mathrm{eV})$ and the Ge electron affinity (4.1 $\mathrm{eV}$ [9]) is $0.4 \mathrm{eV}$. Hence, a barrier height of $0.4 \mathrm{eV}$ would be expected for an $\mathrm{n}-\mathrm{Ge} / \mathrm{Cr}$ contact in the absence of surface states. When a $\mathrm{Ge}_{3} \mathrm{~N}_{4}$ layer is inserted, the barrier seems to be smaller than $0.3 \mathrm{~V}$. This could indicate the existence of a dipole, at the $\mathrm{Ge}_{3} \mathrm{~N}_{4} / \mathrm{Ge}$ interface or the metal $/ \mathrm{Ge}_{3} \mathrm{~N}_{4}$ interface which changes the barrier height. A dipole at the $\mathrm{Ge}_{3} \mathrm{~N}_{4} / \mathrm{Ge}$ interface can be explained by the polarized covalent binding between Ge and $\mathrm{N}$.

In summary, we have investigated the influence of thin intermediate $\mathrm{Ge}_{3} \mathrm{~N}_{4}$ on metal contacts on Ge. Different methods can be used to create such a layer, among them nitrogen plasma exposure, anneal in nitrogen and ammonia atmosphere, resulting in amorphous or (poly)crystalline $\mathrm{Ge}_{3} \mathrm{~N}_{4}$. Where rectifying behavior is observed for all metal contacts on $\mathrm{n}-\mathrm{Ge}$, the insertion of a thin $\mathrm{Ge}_{3} \mathrm{~N}_{4}$ layer leads to Ohmic behavior for $\mathrm{Al}, \mathrm{Cr}$ and Co. Contacts on $\mathrm{n}-\mathrm{Ge}$ of $\mathrm{Au}$ and $\mathrm{Pt}$, which have a higher work function than the previously mentioned metals, still show rectifying behavior when a thin $\mathrm{Ge}_{3} \mathrm{~N}_{4}$ layer is inserted. For $\mathrm{p}-\mathrm{Ge}$ the introduction of a thin 
$\mathrm{Ge}_{3} \mathrm{~N}_{4}$ layer can invoke rectifying behavior instead of Ohmic behavior on bare $\mathrm{p}$-Ge. The experimental results have been explained in terms of (1) the passivation of Ge surface states by $\mathrm{Ge}_{3} \mathrm{~N}_{4}$ and (2) the ionic character of the covalent Ge-N binding which limits the number of $\mathrm{Ge}_{3} \mathrm{~N}_{4}$ surface states. As $\mathrm{Ge}_{3} \mathrm{~N}_{4}$ is a wide bandgap semiconductor, this layer should be kept thin enough to allow electrons to tunnel easily through it. A dipole at the $\mathrm{Ge}_{3} \mathrm{~N}_{4} / \mathrm{Ge}$ interface could have an influence on the barrier height. The high current conduction of metal/ $\mathrm{Ge}_{3} \mathrm{~N}_{4}$ contacts on n-Ge makes dopant implantation to form a tunnel junction superfluous. Furthermore this contact is not stretched into the Ge as for germanides and is therefore shallow. In the case of Ge-based field effect transistors $\mathrm{Ge}_{3} \mathrm{~N}_{4}$ might be used for gate passivation [6] and for contact improvement. Therefore only one $\mathrm{Ge}_{3} \mathrm{~N}_{4}$ deposition step is needed to achieve both goals.

Author R.R. Lieten acknowledges support as a research assistant of the Research Foundation Flanders. 


\section{References}

1. A. Dimoulas, P. Tsipas, A. Sotiropoulos , E. K. Evangelou, Appl. Phys. Lett 89, 252110 (2006)

2. D. Han, Y. Wang, D. Tian, W. Wang, X. Liu, J. Kang, and R. Han. Microelectron. Eng. $82,93(2005)$

3. K. Ikeda, Y. Yamashita, N. Sugiyama, N. Taoka, and S. Takagi, Appl. Phys. Lett. 88, $152115(2006)$

4. T. Maeda, T. Yasuda, M. Nishizawa, N. Miyata, Y. Morita and S. Takagi, Appl. Phys. Lett. 85, 3181 (2004)

5. S. J. Wang, J. W. Chai J. S. Pan and A. C. H. Huan, Appl. Phys. Lett. 89, 022105 (2006)

6. R.R. Lieten, S. Degroote, M. Kuijk, G. Borghs, Appl. Phys. Lett. 91, 222110 (2007)

7. M. Yang, S. J. Wang, Y. P. Feng, G. W. Peng, and Y. Y. Sun, J. Appl. Phys. Lett. 102, 013507 (2007)

8. S. Kurtin, T.C. McGill, and C.A. Mead, Physical Review Letters 22, 1433 (1969)

9. H.J. Kuhr and W. Ranke, Solid State Commun. 61, pp. 285-287 (1987)

10. CRC Handbook of Chemistry and Physics, $85^{\text {th }}$ edition (CRC Press, 2004), pp. 12-130 


\section{Tables}

Table I: Comparison of current density and resistance measured between top and back contact at $-1 \mathrm{~V}$ and between two top contacts at $0.5 \mathrm{~V}$ respectively for different contacts on n-Ge.

Contact Current density Resistance

$\left(\mathrm{A} / \mathrm{cm}^{2}\right) \quad(\mathrm{Ohm})$

\begin{tabular}{lll}
\hline \hline $\mathrm{Al} / \mathrm{Ge}_{3} \mathrm{~N}_{4} / \mathrm{Ge}$ & 13.9 & 16 \\
$\mathrm{Al} / \mathrm{Ge}$ & $5.5 \times 10^{-4}$ & $5.3 \times 10^{5}$ \\
$\mathrm{NiGe} / \mathrm{Ge}$ & $8.9 \times 10^{-3}$ & $4.3 \times 10^{4}$
\end{tabular}


Table II: Metal work function for different metals from ref. 10 and the barrier height calculated from IV measurements. For $\mathrm{Al} / \mathrm{Ge}_{3} \mathrm{~N}_{4}, \mathrm{Cr} / \mathrm{Ge}_{3} \mathrm{~N}_{4}$ and $\mathrm{Co} / \mathrm{Ge}_{3} \mathrm{~N}_{4}$ contacts no barrier was observed and therefore the barrier height is at least smaller than $0.3 \mathrm{eV}$.

\begin{tabular}{lll|l} 
Metal & $\begin{array}{l}\text { Work } \\
\text { function } \\
(\mathbf{e V})\end{array}$ & \multicolumn{2}{c}{ Barrier height (eV) } \\
& $\mathbf{n o} \mathbf{G e}_{\mathbf{3}} \mathbf{N}_{\mathbf{4}}$ & $\mathbf{G e}_{\mathbf{3}} \mathbf{N}_{\mathbf{4}}$ \\
\hline \hline $\mathrm{Al}$ & 4.17 & 0.61 & $<0.3$ \\
$\mathrm{Cr}$ & 4.5 & 0.52 & $<0.3$ \\
$\mathrm{Co}$ & 5.0 & 0.55 & $<0.3$ \\
$\mathrm{Au}$ & 5.38 & 0.59 & 0.51 \\
$\mathrm{Pt}$ & 5.64 & 0.64 & 0.54
\end{tabular}




\section{Figure captions}

Figure 1: IV measurement between a top and backside Ohmic contact on $n-G e(111)$. The top contact consists of $\mathrm{Al} / \mathrm{Ge}_{3} \mathrm{~N}_{4}$ (solid, blue line), $\mathrm{Al}$ (dashed, red line) or NiGe (dotted, black). The insertion of a thin $\mathrm{Ge}_{3} \mathrm{~N}_{4}$ layer clearly changes the contact behavior of $\mathrm{Al}$ from Schottky to Ohmic. $\mathrm{Al} / \mathrm{Ge}_{3} \mathrm{~N}_{4}$ contacts shows much better current conduction than NiGe.

Figure 2: IV measurement between a top metal $/ \mathrm{Ge}_{3} \mathrm{~N}_{4}$ contact and the backside Ohmic contact on $\mathrm{n}-\mathrm{Ge}(111)$. Solid (blue) line for $\mathrm{Al}$, dashed (red) line for $\mathrm{Cr}$, dotted (black) line for Co, dotted-dashed (green) line for Au and dotted-dotted-dashed (brown) line for Pt. $\mathrm{Al}, \mathrm{Cr}$ and $\mathrm{Co}$ form Ohmic contacts when a $\mathrm{Ge}_{3} \mathrm{~N}_{4}$ layer is inserted. Au and Pt stay rectifying.

Figure 3: IV measurement between a top $\mathrm{Al} / \mathrm{Ge}_{3} \mathrm{~N}_{4}$ contact on $\mathrm{p}-\mathrm{Ge}(111)$ and the backside Ohmic contact. The $\mathrm{Al} / \mathrm{Ge}_{3} \mathrm{~N}_{4}$ contact on $\mathrm{p}-\mathrm{Ge}$ is clearly rectifying. 


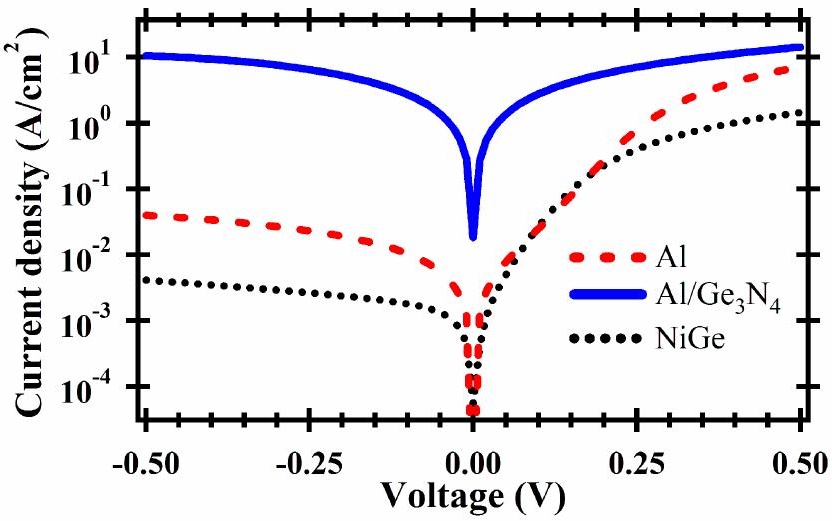




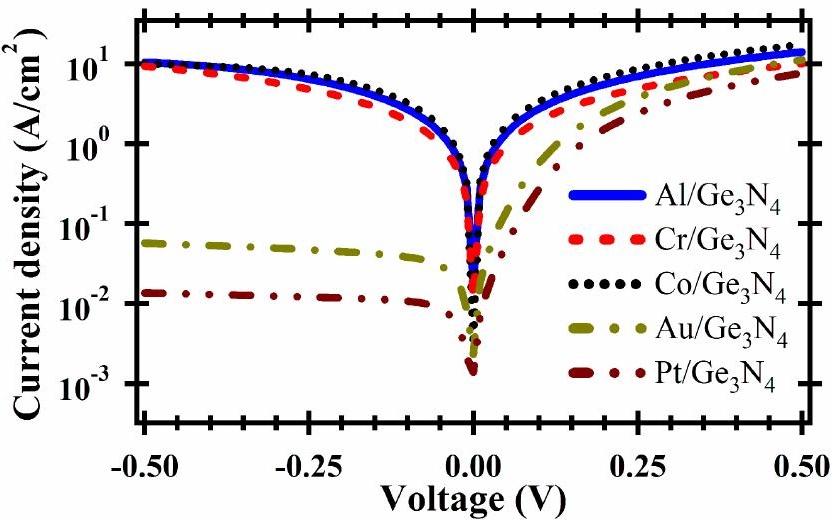




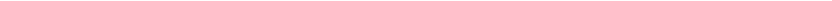

